\title{
Experiencia con el uso de daptomicina en un hospital pediátrico de alta complejidad
}

\author{
Experience with daptomycin in a tertiary pediatric hospital
}

\author{
María T. Rosanova', Norma Sberna'2, Claudia Sarkis', Silvina Ruvinsky', Griselda Berberian ${ }^{1}$ y Rosa Bologna'
}

${ }^{1}$ Servicio de Control Epidemiológico e Infectología. Hospital de Pediatría Juan P. Garrahan. Buenos Aires, Argentina.

${ }^{2}$ Farmacia. Hospital de Pediatría Juan P. Garrahan. Buenos Aires, Argentina.

Los autores firmantes no presentan conflicto de interés.

No hubo financiamiento externo.

Recibido: 19 de julio de 2019 / Aceptado: 9 de diciembre de 2019

\section{Resumen}

Introducción: Vancomicina ha sido considerada como el tratamiento de elección en especial para Staphylococcus aureus resistente a meticilina (SARM); pero su escasa penetración tisular, su toxicidad renal y el requerir monitoreo de su dosis, plantean la necesidad de nuevas alternativas de tratamiento, como daptomicina. Objetivos: Analizar la seguridad y efectividad de daptomicina en niños. Pacientes $y$ Métodos: Se incluyeron, retrospectivamente, niños con infecciones microbiológicamente documentadas, tratados con daptomicina. $R e-$ sultados: Las infecciones más frecuentes fueron endocarditis en 9 $(32 \%)$, sepsis de la comunidad en $4(14 \%)$, bacteriemia en 7 (asociada a catéter en 3 ) (25\%), osteomielitis en $3(10 \%)$, peritonitis asociada a diálisis en $3(10 \%)$ y tromboflebitis supurativa en 2 pacientes (7\%). Staphylococcus aureus resistente a meticilina fue el patógeno más común en 18 pacientes (64\%), Daptomicina fue indicada por el fracaso del tratamiento convencional en $17(61 \%)$, y la toxicidad o intolerancia a vancomicina en 11 pacientes (39\%). La duración media de tratamiento fue de 19 días (RIC 95\% 7-42 días). Cuatro pacientes (14\%) completaron tratamiento ambulatorio. Tuvieron respuesta favorable 22 pacientes (79\%) Se reportaron eventos adversos en tres pacientes: dos elevaciones de creatina-fosfocinasa) y una erupción cutánea grave. Conclusiones: Daptomicina demostró una eficacia y seguridad favorables en esta población pediátrica.

Palabras clave: Daptomicina; infecciones; niños.

\begin{abstract}
Background: Vancomycin has been considered the treatment of choice especially for methicillin-resistant Staphylococcus aureus (MRSA) infections; but its poor tissue penetration, renal toxicity, and requiring of dosages monitoring, raises the need for new treatment alternatives such as daptomycin. Aims: To analyze the safety and effectiveness of daptomycin in children. Methods: Children with microbiologically documented infections treated with daptomycin were retrospectively included. Results: The most frequent infections were endocarditis in $9(32 \%)$, sepsis in $4(14 \%)$, bacteremia in 7 (associated with catheter in 3) $(25 \%)$, osteomyelitis in $3(10 \%)$, peritonitis associated with dialysis in $3(10 \%)$ and suppurative thrombophlebitis in 2 patients $(\mathrm{p})(7 \%)$. Methicillin-resistant Staphylococcus aureus was the most common pathogen in 18 patients (64\%). The indications for daptomycin were due to the failure of conventional treatment in $17(61 \%)$, and the toxicity or intolerance to vancomycin in 11 patients $(39 \%)$. The average duration of treatment was 19 days (95\% ICR 7-42 days). Four patients (14\%) completed outpatient treatment, 22 patients had a favorable response (79\%). Adverse events were reported in 3 patients ( 2 creatinine-phosfo-kinase increase) and in one severe skin rash. Conclusions: Daptomycin demonstrated a favorable efficacy and safety in this pediatric population.

Keywords: Daptomycin; infections; children.
\end{abstract}




\section{Introducción}

$\mathrm{V}$ ancomicina es considerada el antimicrobiano de primera línea en infecciones graves causadas por especies grampositivas sensibles, pero su pobre concentración tisular y su potencial nefrotoxicidad, y el requerir el monitoreo de su dosis para llegar a concentraciones terapéuticas han planteado la necesidad de otras alternativas de tratamiento ${ }^{1,2}$.

A su vez, daptomicina es un antimicrobiano lipopéptido semisintético, concentración dependiente. Tiene actividad bactericida y ha demostrado ser un tratamiento eficaz y bien tolerado, especialmente en adultos con infecciones graves de piel y tejidos blandos incluyendo aquellas causadas por Staphylococcus aureus, independientemente de la susceptibilidad a meticilina ${ }^{1,3}$. Está aprobada en adultos por la Food and Drug Administration (FDA) de Estados Unidos de América y la Agencia Europea de Medicamentos (EMEA) para su uso en infecciones complicadas de la piel y tejidos blandos y en endocarditis infecciosa derecha. A partir del año 2017 fue aprobada para su uso en pediatría.

Teniendo en cuenta su administración una vez al día, el tiempo de infusión corto y un buen perfil de seguridad, daptomicina, podría ser una opción de tratamiento en niños, a pesar de la escasa experiencia descrita en estudios retrospectivos y reportes de $\operatorname{casos}^{1-4}$.

\section{Objetivo}

Evaluar la efectividad clínica y la seguridad de daptomicina en pacientes pediátricos internados con infecciones graves, refractarios o intolerantes a vancomicina.

\section{Material y Métodos}

Estudio, retrospectivo, de historias clínicas de niños hospitalizados que recibieron daptomicina desde el 1 de enero de 2013 al 1 de febrero de 2019.

\section{Criterios de inclusión}

Pacientes mayores de un mes y bajo 18 años de edad, que recibieron al menos una dosis de daptomicina para el tratamiento de infecciones microbiológicamente documentadas por microorganismos grampositivos sensibles al fármaco.

\section{Criterios de exclusión}

Compromiso pulmonar.

El registro de los pacientes se obtuvo de la farmacia del hospital.

\section{Dosis de daptomicina}

6 a $12 \mathrm{mg} / \mathrm{kg} /$ día, administrada una vez por día.

\section{Definiciones}

Fracaso de tratamiento inicial con vancomicina: Hemocultivos persistentemente positivos luego de siete días de tratamiento con concentraciones plasmáticas adecuadas de vancomicina.

Toxicidad e intolerancia a vancomicina: Presencia de nefrotoxicidad, rash intenso u otro efecto adverso que no permitiera continuar con su uso.

Respuesta favorable con daptomicina: Mejoría clínica y /o microbiológica con negativización de cultivos positivos.

Para el análisis de seguridad se incluyó al monitoreo convencional del paciente y la medición semanal de creatina-fosfo-cinasa (sigla en inglés: CPK).

\section{Análisis estadístico}

Las variables numéricas se resumieron como mediana aritmética y rango intercuartílico (RIC). Las variables categóricas fueron informadas como frecuencias absolutas y relativas. El análisis estadístico se realizó utilizando Epi Info versión 6.04

\section{Resultados}

Se incluyeron 28 pacientes. La mediana de edad de los pacientes fue de 45,5 meses (RIC 12-117 meses); 16 (57\%) pacientes eran de sexo masculino.

Dieciocho pacientes (64\%) tenían enfermedades subyacentes.

Las infecciones tratadas con daptomicina fueron: endocarditis (n: 9 pacientes, $32 \%$ ), sepsis de la comunidad (n: 4 pacientes, 14\%), bacteriemia (n: 7 pacientes, $25 \%$ ) (3 de ellas asociadas a catéter), osteomielitis ( $\mathrm{n}: 3$ pacientes, $11 \%$ ), peritonitis asociada a diálisis (n: 3 pacientes, $11 \%$ ) y tromboflebitis supurada (n: 2 pacientes, $7 \%$ ).

Staphylococcus aureus resistente a meticilina (SARM) fue el microorganismo más frecuente (n: 18 pacientes, 64\%), seguido de Staphylococcus coagulasa negativa resistente a meticilina (n: seis pacientes, $21 \%$ ) Enterococcus spp resistente a vancomicina (n: tres pacientes, $11 \%$ ) y $S$. aureus sensible a meticilina (n: un paciente, $4 \%$ ).

Daptomicina se utilizó como terapia de segunda línea en todos los pacientes. Las razones más comunes para la indicación de daptomicina fueron el fracaso del tratamiento inicial (n: 17 pacientes, 61\%), y la toxicidad o intolerancia a vancomicina (n: 11 pacientes, $39 \%$ ). El uso de antimicrobianos concomitantes se informó en 19 pacientes $(68 \%)$, siendo clindamicina, vancomicina y cotrimoxazol los fármacos utilizadas en esos casos.

Nueve pacientes recibieron daptomicina como monoterapia. 
La dosis más frecuentemente indicada fue de $10 \mathrm{mg} /$ $\mathrm{kg} /$ día (r: 6-12 mg/kg/día).

La duración media de la terapia con daptomicina fue de 19 días (RIC 95\% 7-42 días). Cuatro pacientes con endocarditis completaron el tratamiento en forma ambulatoria.

Se observó una respuesta favorable en 22 pacientes $(78,5 \%)$ y seis murieron por causas no relacionadas con el antimicrobiano. Se observaron eventos adversos en tres pacientes (11\%): dos elevaciones de CPK y una erupción cutánea grave. En todos los casos, se suspendió el medicamento con resolución favorable, sin recaídas ni recurrencias (Tablas 1 y 2).

\section{Discusión}

Si bien vancomicina ha sido considerada el antimicrobiano de elección en infecciones graves por cocáceas grampositivas, daptomicina podría ser una opción para infecciones graves por especies grampositivas donde la resistencia emergente a vancomicina, su intolerancia o el fracaso de los tratamientos convencionales dejan pocas alternativas terapéuticas ${ }^{5-10}$.

Las guías de Infectious Diseases Society of America (IDSA) sugieren el uso de daptomicina como agente alternativo en el manejo de infecciones por SARM ${ }^{11}$. El fármaco ha sido estudiado con mayor énfasis en infecciones graves de piel y tejidos blandos y en pacientes adultos $^{6,8-10}$. En pediatría fue utilizada también mayoritariamente en pacientes con infecciones graves de piel y tejidos blandos ${ }^{1-4}$.

En este estudio, la mayor utilización fue en focos endovasculares en que los pacientes persistían con hemocultivos positivos, a pesar de dosificaciones adecuadas, $o$ eran intolerantes a vancomicina.

Las guías IDSA ${ }^{11}$ recomiendan considerar dosis altas de daptomicina en pacientes con bacteriemia persistente por SARM. En otras publicaciones también se sugieren dosis altas en casos de endocarditis, bacteriemia e infecciones osteo-articulares, lo que concuerda con las dosis que debieron ser utilizadas en nuestra experiencia; en algunos pacientes se utilizaron dosis mayores a $10 \mathrm{mg}$ / $\mathrm{kg} /$ día.

Coincidente con otros estudios y con ensayos clínicos en pediatría ${ }^{1-4}$, se observó que un alto porcentaje de los pacientes tuvo una evolución favorable, con resolución clínica y microbiológica de la infección.

Si bien en una publicación ${ }^{5}$ se describe el uso de daptomicina en neumonía, en nuestro caso la presencia de foco pulmonar fue un criterio de exclusión, ya que este fármaco es inhibido por el surfactante pulmonar, provocando el fallo clínico subsecuente.

Dada la posibilidad de administrar daptomicina una
Tabla 1. Tipo de infecciones y microorganismos causales

\begin{tabular}{|c|c|c|}
\hline Tipo de infección & n (\%) & Etiología \\
\hline Endocarditis & $9(32 \%)$ & 6 SARM, 2 SCN y 1 SASM \\
\hline Sepsis de la comunidad & $4(14 \%)$ & 4 SARM \\
\hline Bacteriemia & $7(25 \%)$ & 3 SARM-3 SCN- 1 ERV \\
\hline Osteomielitis & $3(10 \%)$ & 3 SARM \\
\hline Peritonitis asociada a diálisis & $3(10 \%)$ & 2 ERV y 1 SCN \\
\hline Tromboflebitis supurativa & $2(7 \%)$ & 2 SARM \\
\hline \multicolumn{3}{|c|}{$\begin{array}{l}\text { SARM: Staphylococcus aureus resistente a meticilina; SAMS: } \\
\text { Staphylococcus aureus sensible a meticilina SCN: Staphylo- } \\
\text { coccus coagulasa negativa; ERV: Enterococcus spp resistente } \\
\text { a vancomicina. }\end{array}$} \\
\hline
\end{tabular}

Tabla 2. Dosis de daptomicina empleada y evolución de los pacientes ( $\mathrm{n}: 28 \mathrm{p}$ ) Dosis diaria de daptomicina

Días de administración Mediana $10 \mathrm{mg} / \mathrm{kg} /$ día (r: 6-12 mg/kg/día)

Evolución favorable Mediana de 19 d (RIC: 7-42 d) 22 p $(79 \%)$

vez por día, completamos el tratamiento en forma ambulatoria en pacientes cuidadosamente seleccionados, lo que favoreció el confort y la externalización precoz, a pesar de no encontrar prescripciones de esta modalidad en la literatura médica consultada.

Con respecto a los efectos adversos de daptomicina, el aumento de CPK ha sido notificado en pacientes adultos y niños y ha sido infrecuente la suspensión del fármaco por este motivo, tal como fue en nuestra serie ${ }^{2-4}$.

Las concentraciones plasmáticas de CPK deben controlarse semanalmente o, con mayor frecuencia, en pacientes con mialgias, miopatías o insuficiencia renal concomitante, o cuando se administran conjuntamente con fármacos asociados con el incremento de $\mathrm{CPK}^{13}$.

Algunas limitaciones en nuestro análisis son inherentes al diseño retrospectivo, el tratarse de un solo centro de alta complejidad, y no haberse realizado un estudio randomizado. Otra limitante es que la mayoría de los pacientes recibiera antimicrobianos concomitantes; por lo tanto, no se puede atribuir el éxito del tratamiento exclusivamente a daptomicina.

\section{Conclusiones}

En esta serie, daptomicina, sola o combinada con otros antimicrobianos, fue una alternativa de segunda línea efectiva y segura para infecciones graves producidas por microorganismos grampositivos. 


\section{Referencias bibliográficas}

1.- Namtu K, Crain J, Messina A, Dumois J, Berman D M. Clinical experience with daptomycin in pediatrics. Pharmacotherapy 2017; 37 (1):105-8. doi: 10.1002/phar.1872.

2.- Syrongiannopoulos G, Michoula A, Petinaki E and Grivea L. Daptomycin use in children: Experience with various types of infection and age groups. Pediatr Infect Dis J 2017; 36: 962 6. doi: 10.1097/INF.0000000000001629.

3.- Bradley J, Glasser C, Patino H, Arnold SR, Arrieta A, Congeni B, et al. Daptomycin for complicated skin infections: a randomized trial. pediatrics 2017; 139: e20162477. doi: 10.1542/ peds.2016-2477.

4.- Arrieta AC, Bradley JS, Popejoy M W, Bensaci M, Grandhi A, Bokesch P, et al. Randomized multicenter study comparing safety and efficacy of daptomycin versus standard-of-care in pediatric patients with staphylococcal bacteremia. Pediatr Infect Dis J 2018; 37: 893-900. doi: 10.1097/ INF.00000000001926.

5.- Pertel PE, Bernardo P, Fogarty C, Matthews $\mathrm{P}$, Northland R, Benvenuto M, et al. Effects of prior effective therapy on the efficacy of daptomycin and ceftriaxone for the treatment of community-acquired pneumonia. Clin Infect Dis 2008; 46: 1142-51 doi: 10.1086/533441.

6.- Arbeit R D, Maki D, Tally F P, Campanaro E, Eisenstein B. The safety and efficacy of daptomycin for the treatment of complicated skin and skin-structure infections. Clin Infect Dis 2004; 38:1673-81. doi: 10.1086/420818.

7.- Fowler V G Jr, Boucher H W, Corey G R, Abrutyn E, Karchmer A W, Rupp M E, et al. Daptomycin versus standard therapy for bacteremia and endocarditis caused by Staphylococcus aureus. N Engl J Med 2006; 355: 653-65. doi:10.1056/NEJMoa053783.

8.- Pertel P E, Eisenstein B I, Link A S, Donfrid B. The efficacy and safety of daptomycin vs. vancomycin for the treatment of cellulitis and erysipelas. Int J Clin Pract 2009; 63:368-75. doi: 10.1111/j.1742-1241.2008.01988.x.

9.- Konychev A, Heep M, Moritz R K, Kreuter A, Shulutko A, Fierlbeck G, et al. Safety and efficacy of daptomycin as first-line treatment for complicated skin and soft tissue infections in elderly patients: an open-label, multicentre, randomized Phase IIIb trial. Drugs Aging 2013; 30: 829-36. doi:10.1007/s40266-013-0114-8.

10.- Aikawa N, Kusachi S, Mikamo,H, Takesue Y, Watanabe S, Tanaka Y, et al. Efficacy and safety of intravenous daptomycin in Japanese patients with skin and soft tissue infections. J Infect Chemother 2013; 19: 447-55. doi.org/10.1007/ s10156-012-0501-9.

11.- Liu C, Bayer A, Cosgrove S E Daum R S, Fridkin S K, Gorwitz R J, et al. Clinical practice guidelines by the Infectious Diseases Society of America for the treatment of methicillin-resistant Staphylococcus aureus infections in children and adults. Clin Infect Dis 2011; 52: e:18-e55. doi: 10.1093/cid/ciq146.

12.- González-Ruiz A, R Seaton A, Hamed K. Daptomycin: an evidence-based review of its role in the treatment of Gram-positive infections. Infect Drug Resist. 2016; 9: 47-58. doi: 10.2147/IDR.S99046.

13.- D'Avolio A, Pensi D, Baietto L, Pacini G, Di Perri G, De Rosa F G. Daptomycin pharmacokinetics and pharmacodynamics in septic and critically ill patients. Drugs 2016; 76 (12): 1161-74. doi: 10.1007/s40265-0160610-3. 